\title{
Randomized Clinical Trial of Balloon Occlusion versus Conventional Microcatheter Prostatic Artery Embolization for Benign Prostatic Hyperplasia
}

\author{
Tiago Bilhim, PhD, MD, EBIR, Nuno Vasco Costa, MD, Daniel Torres, MD, \\ João Pisco, MD, PhD, Sandra Carmo, MSc, and \\ António G. Oliveira, MD, PhD
}

\begin{abstract}
Purpose: To compare balloon occlusion prostatic artery embolization (bPAE) with conventional microcatheter PAE (cPAE).

Materials and Methods: In this single-center trial, between November 2017 and November 2018, 89 patients with symptomatic benign prostatic hyperplasia were randomly assigned to $\operatorname{cPAE}(n=43)$ or bPAE $(n=46)$. All patients received embolization with 300 $500 \mu \mathrm{m}$ Embosphere microspheres and were evaluated before and 1 and 6 months after PAE. Primary outcome measure was change from baseline in International Prostate Symptom Score (IPSS). Student $t$ test was used for between-group comparisons of change from baseline, and paired $t$ test was used for within-group comparisons.

Results: At baseline, groups were identical $(P>.05)$. Unilateral PAE was performed in 4 patients receiving CPAE and 3 patients receiving bPAE $(9.30 \%$ and $6.52 \%, P=.708)$. Procedural and fluoroscopy times, dose area product, air kerma, embolic volume, and mean prostate-specific antigen (PSA) 24 hours after PAE did not differ between groups $(P>.05)$. Coils were used in 6 patients receiving cPAE and 4 patients receiving bPAE $(14.0 \%$ and $8.70 \%, P=.51)$. Assessments at 6 months after PAE showed mean IPSS reduction was $7.58 \pm 6.88$ after cPAE and $8.30 \pm 8.12$ after bPAE $(P=.65)$; mean prostate volume reduction was $21.9 \mathrm{~cm}^{3} \pm 51.6(18.2 \%)$ after cPAE and $6.15 \mathrm{~cm}^{3} \pm 14.6(7.3 \%)$ after bPAE $(P=.05)$; mean PSA reduction was $0.9 \mathrm{ng} / \mathrm{mL} \pm 2.22$ after cPAE and $0.22 \mathrm{ng} / \mathrm{mL} \pm 1.65$ after bPAE $(P=.10)$. Penile skin lesions $(\mathrm{n}=3)$ and rectal bleeding $(\mathrm{n}=2)$ were documented only in patients receiving cPAE $(11.9 \%, P=.01)$. No major adverse events occurred.
\end{abstract}

Conclusions: bPAE is as effective as cPAE in treating benign prostatic hyperplasia with a potential to reduce nontarget embolization.

\section{ABBREVIATIONS}

$\mathrm{bPAE}=$ balloon occlusion prostatic artery embolization, $\mathrm{cPAE}=$ conventional microcatheter prostatic artery embolization, IPSS $=$ International Prostate Symptom Score, LUTS = lower urinary tract symptoms, PAE = prostatic artery embolization, PSA $=$ prostatespecific antigen

Prostatic artery embolization (PAE) has been shown to be safe and effective for the relief of lower urinary tract symptoms (LUTS) associated with benign prostatic hyperplasia (1). However, $20 \%-25 \%$ of patients experience clinical failure after PAE (2). The vast majority of these patients never respond to PAE (nonresponders); a minority
From the Interventional Radiology Unit (T.B., N.V.C., D.T., J.P., S.C.), Saint Louis Hospital, R. Luz Soriano 182, Lisbon 1200-249, Portugal; Interventional Radiology Unit (T.B., N.V.C., D.T.), Radiology (S.C.), Centro Hospitalar Universitário de Lisboa Central, Lisbon, Portugal; Nova Medical School (T.B., N.V.C., D.T.), Faculdade de Ciências Médicas, Universidade Nova de Lisboa, Lisbon, Portugal; and Pharmacy Department (A.G.O.), Universidade Federal do Rio Grande do Norte, Natal, Rio Grande do Norte, Brazil. Received March 4, 2019; final revision received June 25, 2019; accepted June 26, 2019. Address correspondence to T.B.; E-mail: tiagobilhim@hotmail.com

T.B. is a paid consultant for Merit Medical Systems, Inc (South Jordan, Utah), Terumo Corporation (Tokyo, Japan), and Philips Healthcare (Best, The
Netherlands), an advisory board member for Merit Medical Systems, Inc, and is a stockholder in Embolx, Inc (Sunnyvale, California). N.V.C. is a paid consultant for Philips Healthcare. J.P. is a paid consultant for Merit Medical Systems, Inc and is a stockholder in Embolx, Inc. None of the other authors have identified a conflict of interest.

(c) SIR, 2019

J Vasc Interv Radiol 2019; 30:1798-1806

https://doi.org/10.1016/j.jvir.2019.06.019 
of patients experience initial symptomatic relief with relapse of LUTS at midterm and long-term follow-up (3). It is currently accepted that bilateral PAE is more effective than unilateral PAE (3). Other technical aspects of PAE have been suggested to contribute to the success of the procedure. Specifically, wedged embolization, placing the microcatheter tip distally inside the intraprostatic vasculature, may have advantages over free-flow embolization from the proximal prostatic artery trunk (4). Particle size has yielded conflicting results, and more evidence is required concerning the potential beneficial effects of using smaller sized particles in PAE (5-9). Balloon occlusion PAE (bPAE) is a safe and effective procedure that potentially prevents the reflux of embolic agents to the pelvic branches and may minimize untargeted embolization to surrounding organs by creating a negative pressure inside the prostatic vasculature with reversal of blood flow through the arterial anastomoses (10). The purpose of this study was to compare the safety and efficacy of bPAE versus conventional microcatheter PAE (cPAE).

\section{MATERIALS AND METHODS}

\section{Trial Design and Participants}

This single-center prospective randomized single-blind clinical trial was conducted between November 2017 and November 2018. The study was approved by the institutional review board, and all patients gave informed written consent. Inclusion criteria were $(a)$ age $>40$ years old; $(b)$ prostate volume $>30 \mathrm{~cm}^{3}$; (c) LUTS secondary to benign prostatic hyperplasia with an International Prostate Symptom Score (IPSS) $>18$ points and/or quality of life related to LUTS $>3$ points; $(d)$ refractoriness to medical therapy for $>6$ months or refusal of medical therapy. Exclusion criteria were $(a)$ acute urinary retention; $(b)$ prostate or bladder malignancy; (c) bladder diverticula $>5 \mathrm{~cm}$ or stone $>2 \mathrm{~cm}$; (d) chronic renal failure; (e) acute urinary tract infection; $(f)$ patient unable to provide follow-up data; $(g)$ embolization with other embolic agents or particle sizes; $(h)$ patient participation in another clinical trial; (i) previous PAE procedure $(2,3)$.

\section{Interventions}

All included patients received PAE from a unilateral retrograde femoral approach. A 5-F Pisco prostate catheter (Merit Medical Systems, Inc, South Jordan, Utah) was used to catheterize both internal iliac arteries after reforming the catheter with the Waltman loop in the aortic bifurcation. For selective catheterization of the prostatic arteries, the Maestro 2.4-F, Swan Neck-tip microcatheter (Merit Medical Systems, Inc) was used in the cPAE group, and the Sniper 2.2-F, straight-tip microcatheter (Embolx, Inc, Sunnyvale, California) was used in the bPAE group. The micro-guide wires used with the microcatheters were the 0.016 inch hydrophilic GT double-angled wire (Terumo Corporation, Tokyo, Japan) or the 0.018 inch hydrophilic shapeable tip wire
(Tenor; Merit Medical Systems, Inc). Computed tomography (CT) angiography was used to map the pelvic arterial anatomy before PAE in all patients (11). All PAE procedures were performed with an Allura FD20 angiography unit (Philips Healthcare, Best, The Netherlands). On reaching the prostatic arteries, either cone-beam CT or digital subtraction angiography was performed (XperCT; Philips Healthcare). The cone-beam CT protocol consisted of pump injection of nondiluted iodine contrast agent ioversol $350 \mathrm{mg}$ iodine $/ \mathrm{mL}$ (Optiray 350; Covidien, Dublin, Ireland), $6 \mathrm{~mL}$ at $0.5 \mathrm{~mL} / \mathrm{s}$, arrival time of 1.5 seconds, 10 -second rotational scan of $180^{\circ}$ at $18^{\circ}$ rotation per second, image acquisition every $0.5^{\circ}$, source power of $125 \mathrm{kVp}$, and 316 matrix images $(512 \times 512$ voxels $)$. The digital subtraction angiography protocol was in posteroanterior view with the same contrast agent and with pump injection of $5 \mathrm{~mL}$ at $2 \mathrm{~mL} / \mathrm{s}$. The microcatheter was advanced as distally as possible inside the prostatic arteries into the intraprostatic branches in the CPAE group, and a wedged embolization was performed allowing reflux toward the main prostatic artery trunk (Fig 1). The endpoint was set when reflux of embolic material approached the prostatic artery origin. In the bPAE group, the microcatheter was placed in the middle third of the prostatic artery (Fig 2). The balloon was inflated with $0.1-0.2 \mathrm{~mL}$ of contrast agent under fluoroscopy. Selective prostatic cone-beam CT angiography or digital subtraction angiography, using the above-described parameters, was performed after inflating the balloon. Embolization was then initiated, and the endpoint was reached when the embolic injection started to push the balloon backward inside the prostatic artery. If arterial anastomoses were detected after selective angiography of the prostatic arteries, coil embolization was performed to prevent off-target embolization. Coils were also used to redirect flow into the prostatic artery when selective catheterization was not possible. After contrast injection and before embolization, 100-200 $\mu \mathrm{g}$ of nitroglycerin (Hospira UK Limited, Hurley, Maidenhead, United Kingdom) was injected through the microcatheter in both groups. All patients received embolization with 300$500 \mu \mathrm{m}$ Embosphere microspheres (Merit Medical Systems, Inc). A stable suspension of microspheres was obtained by filling the syringe vial with contrast agent and saline up to the limit of $20 \mathrm{~mL}$. All procedures were performed by 3 interventional radiologists with 10 (T.B.), 5 (N.V.C.), and 3 (D.T.) years of experience in PAE. Patient medication protocol before and after embolization was the same in both groups and was described previously (2).

\section{Outcomes}

Patients were evaluated before PAE, 1 month after PAE, and 6 months after PAE. The parameters assessed were IPSS/ quality of life and International Index of Erectile Function scores, prostate volume measured with transrectal ultrasound using the ellipsoid formula, prostate-specific antigen (PSA), uroflowmetry measuring peak flow rate, and postvoid residual. The primary outcome was the change from 


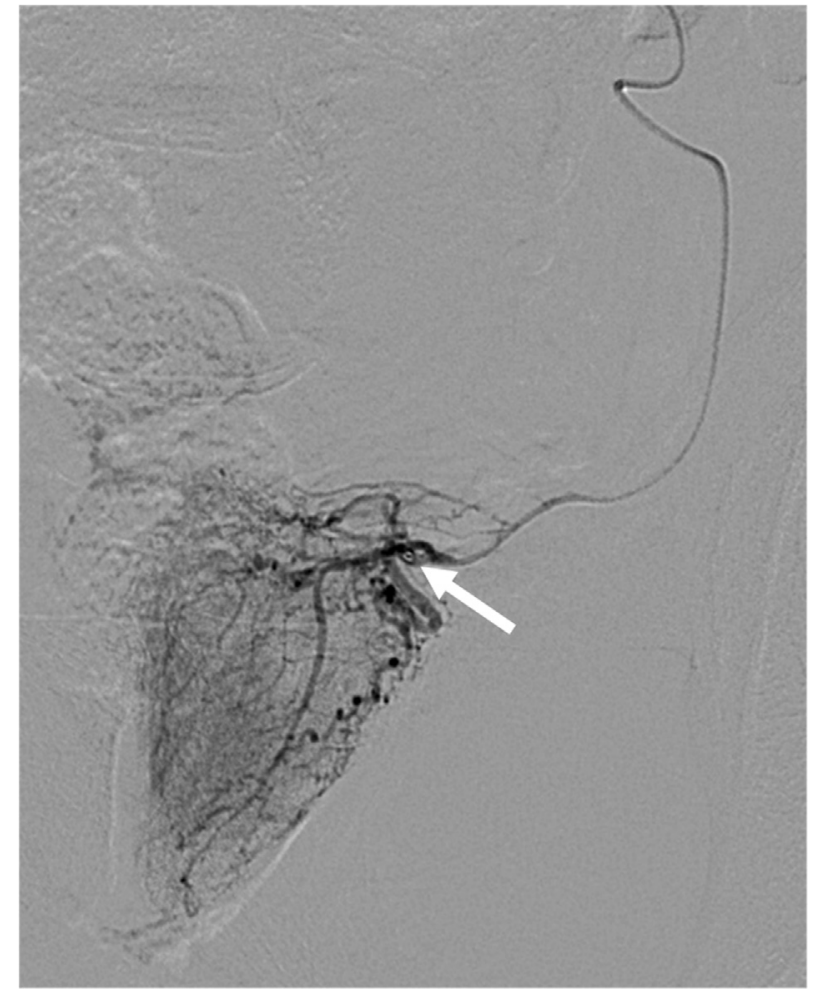

Figure 1. Digital subtraction angiography in posteroanterior view with microcatheter tip (arrow) advanced as distally as possible inside the left prostatic artery into the intraprostatic branches.

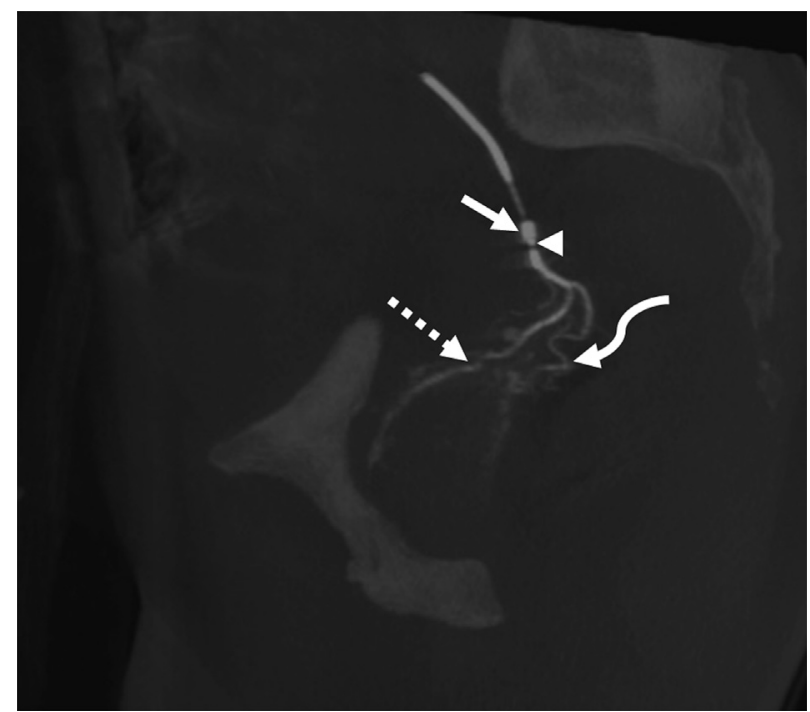

Figure 2. Oblique sagittal reformat of cone-beam CT depicting the balloon occlusion microcatheter tip placed in the middle third of the left prostatic artery (arrowhead). Note the inflated occlusion balloon (arrow) proximal to the microcatheter tip. Central gland prostatic artery branch (dashed arrow) and peripheral gland prostatic branch (curved arrow) are also seen.

baseline in IPSS. Secondary outcome variables were related to the PAE procedure: procedural and fluoroscopy times, dose area product, air kerma, embolic volume, need to use coils to block anastomoses, and PSA 24 hours after PAE.
Post hoc analysis also considered the correlation between baseline prostatic medication, volume of embolic agents used, PSA 24 hours after PAE, and clinical outcomes. Adverse events were classified according to Society of Interventional Radiology (SIR) criteria (12) and prospectively collected using a previously published form (5). No changes to trial outcomes or methodology were made during the trial.

\section{Sample Size and Randomization}

Data from a previous series of 300 patients estimated the standard deviation of the change from baseline of the IPSS score at 6 months as approximately 7 points $(2,3)$. In the absence of published data on the expected difference between cPAE and bPAE, the clinical trial was powered to $70 \%$ to expose differences between groups in the change from baseline of the IPSS score at 6 months of 3.5 points, which is generally considered as the minimum for clinically significant differences, resulting in a sample size of 50 patients per group, for a significance level of 5\% with a 2sided test. Patients were allocated to groups by simple 1:1 randomization, immediately before the procedure and masked from the operators. Patients, but not the operators, were blinded to the technique being used.

\section{Statistical Methods}

The trial was analyzed according to the intention-to-treat principle, and all randomly assigned patients were included in the efficacy analysis set. Missing data were imputed using the last observation carried forward method. Study variables are presented descriptively as mean \pm SD or as frequency and percentage. Student $t$ test was used for between-group comparisons of the change from baseline in the primary and secondary efficacy variables, and the paired $t$ test was used for within-group comparisons. Proportions were compared between groups with Fisher exact test. For subgroup analyses, multiple linear regression was used to test the covariate by treatment interaction on the change from baseline in IPSS. All tests were bilateral, and differences with $\mathrm{P}<.05$ were considered statistically significant. Stata 13 (StataCorp LLC, College Station, Texas) was used for statistical analysis.

\section{RESULTS}

Of 176 patients evaluated for inclusion in the trial, 87 patients were excluded for the following reasons (Fig 3): foreigner with limited availability to provide follow-up data $(\mathrm{n}=37)$, diagnosis of prostate cancer $(\mathrm{n}=11)$, PAE performed with different embolic agents and/or sizes $(\mathrm{n}=$ $9)$, previous PAE $(\mathrm{n}=22)$, and acute urinary retention $(\mathrm{n}=$ 8). Therefore, 89 patients were randomly assigned to PAE with a conventional microcatheter (Maestro; cPAE group, $\mathrm{n}=43$ ) or a balloon occlusion microcatheter (Sniper; bPAE group, $n=46$ ). Mean patient age was 67.3 years \pm 8.02 in the cPAE group and 65.8 years \pm 7.93 in the bPAE group 


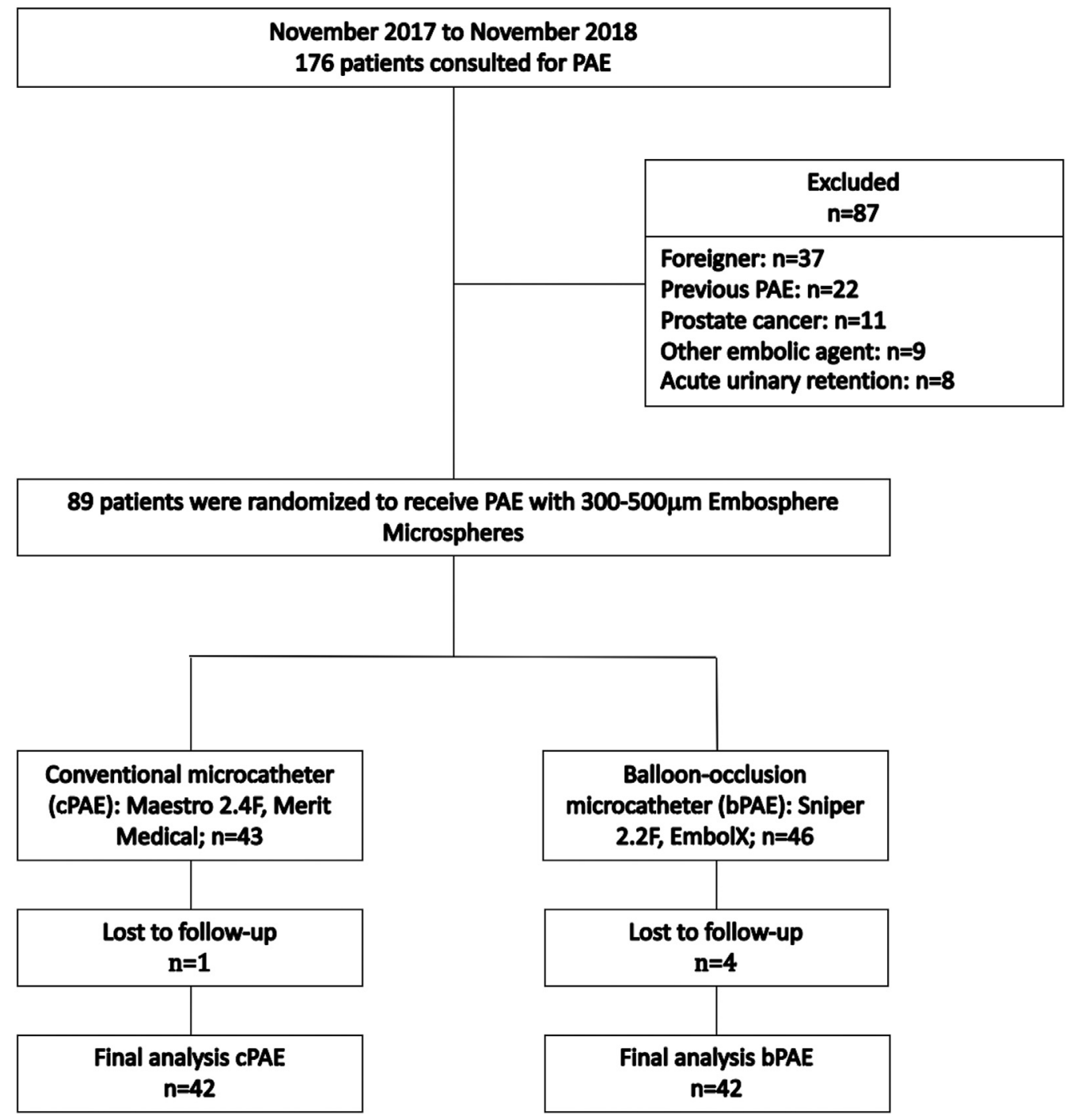

Figure 3. Participant flow diagram.

$(P=.38)$. There were no appreciable differences between groups in the efficacy variables. Overall, 64 patients (71.9\%) were taking prostatic medication: $\alpha$-blockers (46 patients; 51.7\%), 5 $\alpha$-reductase inhibitors (4 patients; $4.49 \%$ ), or both (14 patients; $15.7 \%$ ) with no significant differences between groups $(P=.51)$. Five patients dropped out of the study within 1 month after embolization (1 patient in the cPAE group and 4 patients in the bPAE group).

Unilateral PAE was performed in 4 patients in the cPAE group and 3 patients in the bPAE group $(9.30 \%$ and $6.52 \%$, respectively, $P=.708$ ) owing to iliac tortuosity, pelvic atherosclerosis, or angulated origins of the prostatic artery from the superior vesical artery. No patients crossed over from bPAE to cPAE or vice versa. Coils were used in 6 patients in the cPAE group and 4 patients in the bPAE group $(14.0 \%$ and $8.70 \%, P=.51)$. The microcatheter in the bPAE group failed to reach distally inside the prostatic artery in 3 $(6.52 \%)$ of 46 patients (superior vesical artery in 2 patients and obturator artery in 1 patient), and coils were placed to redirect flow. The microcatheter in the cPAE group failed to reach distally inside the prostatic artery in $1(2.32 \%)$ of 43 patients (superior vesical artery), and coils were placed to redirect flow $(P=.17)$. Coils were used to block rectal anastomoses in 1 patient in the bPAE group. In the cPAE group, coils were placed to block penile anastomosis in 4 patients and rectal anastomosis in 1 patient. Additional details of the procedure for both groups are presented in Table 1 and Figures 4a-e, 5a, b, and 6a-c.

All efficacy variables improved in both groups with statistical significance with the exception of International Index of Erectile Function, which remained constant in both groups, and PSA, which decreased significantly in the cPAE group $(P=.01)$, but not in the bPAE group $(P=.38)$. Table 2 shows the within-group analysis of the efficacy variables.

When comparing between groups, the mean change from baseline of the primary efficacy variable IPSS was -7.58 points \pm 6.88 and -8.30 points \pm 8.12 in the cPAE and 
Table 1. Procedure Variables

\begin{tabular}{|c|c|c|c|c|c|}
\hline \multirow[t]{2}{*}{ Variable } & \multirow[t]{2}{*}{ cPAE $(n=43)$, Mean (SD) } & \multirow[t]{2}{*}{ bPAE $(n=46)$, Mean (SD) } & \multicolumn{2}{|c|}{ Difference } & \multirow[t]{2}{*}{$P$ Value } \\
\hline & & & Mean & $95 \% \mathrm{Cl}$ & \\
\hline Procedural time, $\min$ & $71.2(26.8)$ & $72.8(22.5)$ & -1.66 & -12.1 to 8.73 & .751 \\
\hline Dose area product, $\mathrm{mGy} / \mathrm{cm}^{2}$ & $281,343(178,039)$ & $294,730(120,972)$ & $-13,387$ & $-97,186$ to 40,209 & .412 \\
\hline Air kerma, mGy & $2,117(1,588)$ & $2,065(846)$ & 52 & $-15,301$ to 33,584 & .331 \\
\hline Embolic volume, $\mathrm{mL}$ & $11.4(3.63)$ & $12.0(3.88)$ & -0.58 & -2.17 to 1.01 & .470 \\
\hline
\end{tabular}

$\mathrm{bPAE}=$ balloon occlusion prostatic artery embolization; $\mathrm{Cl}=$ confidence interval; $\mathrm{cPAE}=$ conventional microcatheter prostatic artery embolization; PSA = prostatic-specific antigen.
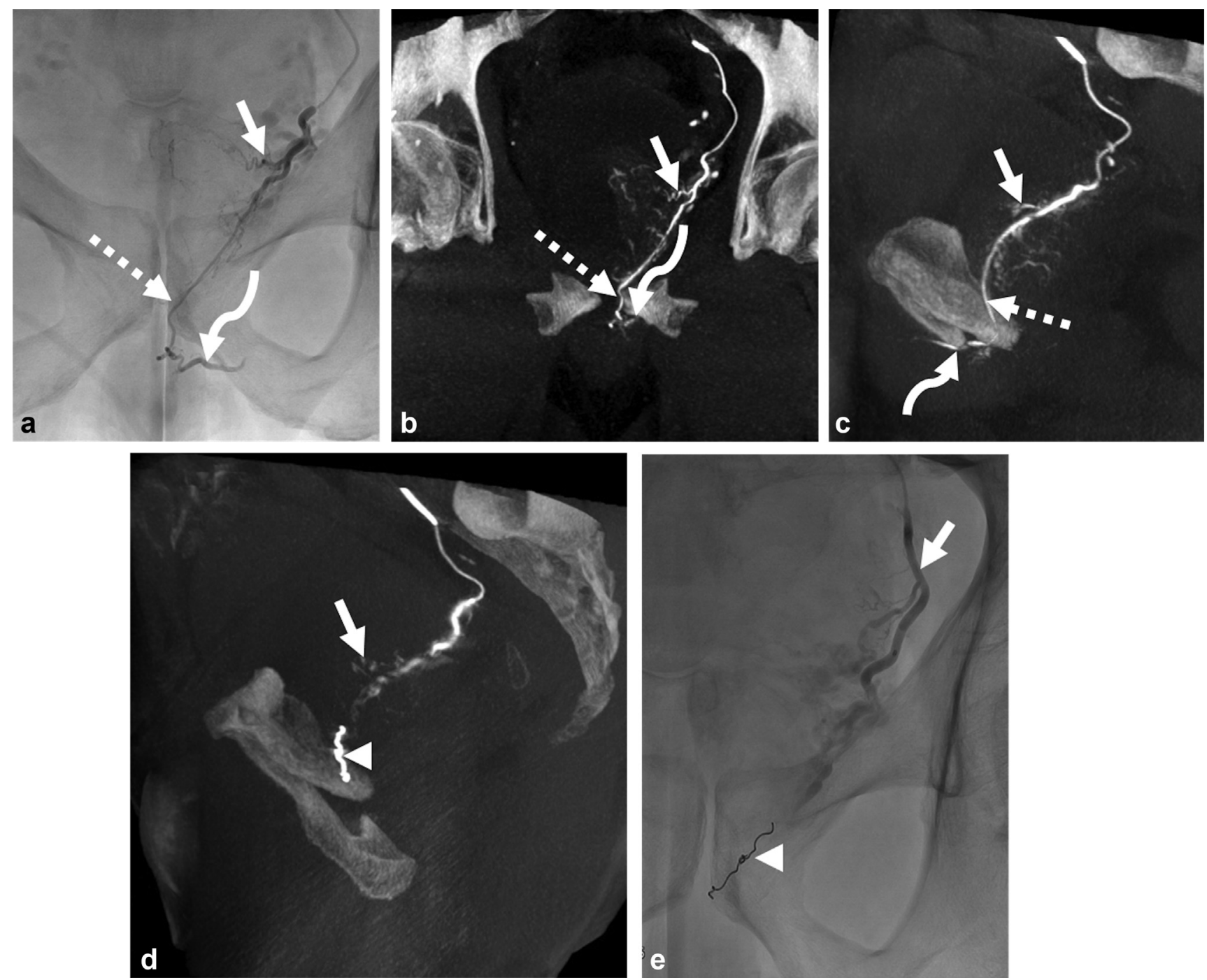

Figure 4. Coil-assisted left PAE with conventional microcatheter. (a) Selective left prostatic artery digital subtraction angiography in posteroanterior view depicting the left prostate gland supplying branch (arrow) and a large anastomosis (dashed arrow) to the penile artery (curved arrow). (b) Oblique coronal cone-beam CT reformat depicting the left prostate gland supplying branch (arrow) and a large anastomosis (dashed arrow) to the penile artery (curved arrow). (c) Oblique sagittal reformat of cone-beam CT depicting the left prostate gland supplying branch (arrow) and a large anastomosis (dashed arrow) to the penile artery (curved arrow). (d) Oblique sagittal reformat of cone-beam CT depicting the left prostate gland supplying branch (arrow) after coil embolization of the penile anastomosis (arrowhead). (e) Digital subtraction angiography in posteroanterior view after embolization of the left prostatic artery depicting the coils (arrowhead) and reflux of embolic material almost reaching the prostatic artery origin (arrow). 

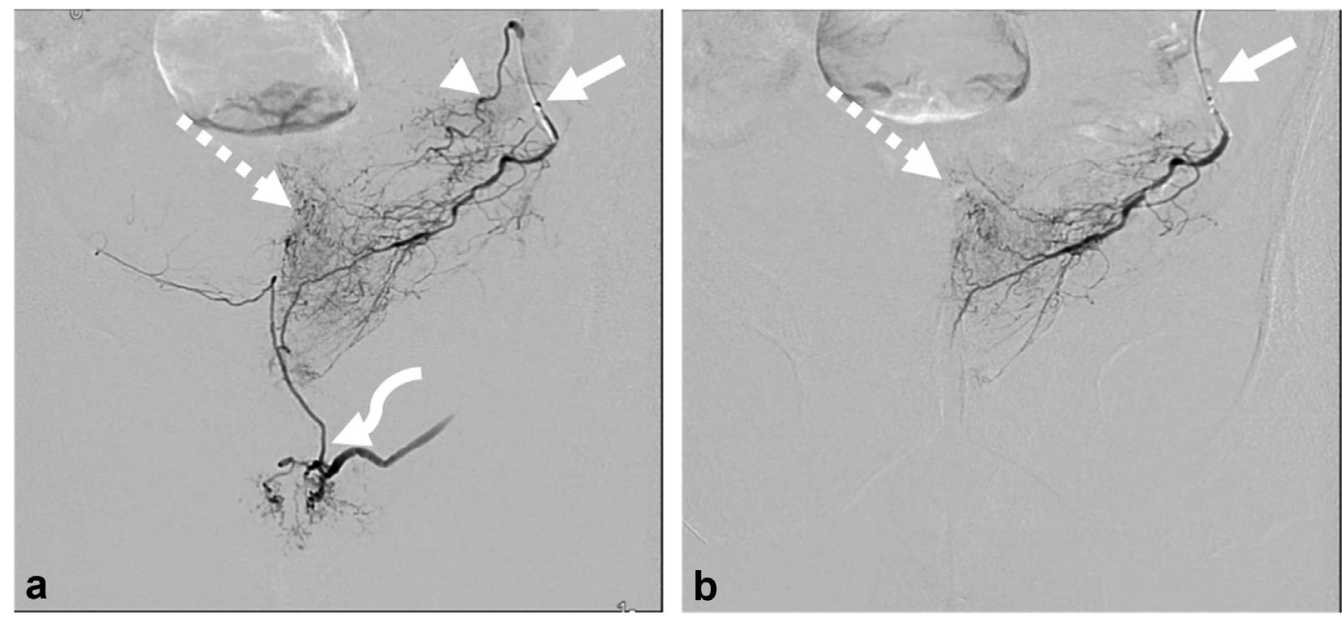

Figure 5. Balloon occlusion left PAE. (a) Selective left prostatic artery digital subtraction angiography in posteroanterior view with the occlusion balloon not inflated (arrow) depicting the left prostate gland opacification (dashed arrow) and a large anastomosis to the penile artery (curved arrow) and to the bladder base (arrowhead). (b) Selective left prostatic artery digital subtraction angiography in posteroanterior view with the occlusion balloon inflated (arrow) depicting the left prostate gland opacification (dashed arrow) without visible anastomosis owing to reversal of blood flow. Embolization was performed without the need for coil placement.

bPAE groups, respectively. The difference between the groups was not statistically significant $(95 \%$ confidence interval -2.46 to 3.90 points, $P=.65$ ). The reduction in prostate volume was $-21.9 \mathrm{~cm}^{3} \pm 51.6$ and $-6.15 \mathrm{~cm}^{3} \pm$ 14.6 in the cPAE and bPAE groups, respectively $(P=.05)$. In relative terms, the average percent decrease in prostate volume was $18.2 \%$ in patients receiving cPAE and $7.30 \%$ in patients receiving bPAE. There were no statistically significant differences between groups for all the other outcome variables (Table 3).

Quantification of PSA levels 24 hours after PAE was available for 30 patients in the cPAE group and 31 patients in the bPAE group. The mean values were $104.2 \mathrm{ng} / \mathrm{mL} \pm$ 109.3 in the cPAE group and $82.2 \mathrm{ng} / \mathrm{mL} \pm 94.1$ in the bPAE group $(P=.40)$. The average percent increase of PSA 24 hours after PAE from baseline was 3,453\% \pm $3,344 \%$ in the cPAE group and $2,935 \% \pm 2,998 \%$ in the bPAE group $(P=.53)$. Subgroup analyses did not show statistically significant interactions between IPSS reduction after PAE and total embolic volume or baseline prostate volume $(P>.99)$, PSA 24 hours after PAE $(P=$ $.46)$, increase in PSA 24 hours after PAE $(P=.14)$, and previous medication with $\alpha$-blockers $(P=.62)$. For previous treatment with $5 \alpha$-reductase inhibitors, there was evidence of a minor interaction with $\operatorname{PAE}(P=.04)$ : for patients in the cPAE group previously treated with that class of drugs, the average decrease from baseline in IPSS was 11.8 points \pm 5.04 versus 5.33 points \pm 4.18 in patients without previous treatment.

Intraprostatic arterial rupture resulting from overpressured embolization occurred in 4 procedures, 2 cases in the bPAE group and 2 cases in the cPAE group. Balloon rupture occurred in 5 procedures, which required the use of a new balloon occlusion microcatheter. There was no statistically significant difference in the average maximum pain reported within 24 hours after the procedure $(0.74 \pm 1.31$ and $0.93 \pm$
1.70 in cPAE and bPAE groups, respectively, $P=.56$ ). Minor adverse events were reported in 23 patients (53.5\%) in the cPAE group and 26 patients $(56.5 \%)$ in the bPAE group $(P=.83)$. One patient in the cPAE group had acute urinary retention in the first month following PAE, which resolved with medical therapy. The other adverse events were mild and resolved without therapy. No major adverse events, such as impotence or urinary incontinence, were noted. Table 4 shows the frequency of adverse events after PAE. Penile skin lesions $(\mathrm{n}=3 ; 7.0 \%)$ and rectal bleeding $(\mathrm{n}=2 ; 4.7 \%)$ were documented only in patients in the cPAE group $(11.7 \%, P=.01)$.

\section{DISCUSSION}

The present trial was intended to prospectively establish whether bPAE would be beneficial over cPAE. The main outcome measure was relief of LUTS as measured by IPSS reduction after PAE, which showed no significant differences between the 2 groups. bPAE relies on the potential to prevent reflux and to reverse blood flow in the anastomoses between the prostate and surrounding organs. Thus, it would be reasonable to expect fewer adverse events in bPAE and less need for protective coil blockage of the prostatic anastomoses. These assumptions were not proven here, as the overall rate of adverse events was not significantly different between the 2 groups. However, penile skin lesions $(\mathrm{n}=3 ; 7.0 \%)$ and rectal bleeding $(\mathrm{n}=2 ; 4.7 \%)$, which can be due to nontarget embolization, were significantly more frequent in the cPAE group, with no documented cases in the bPAE group.

These observations are probably clinically relevant, as they represent indirect measures of nontarget embolization. Rectal bleeding has been reported in $3 \%-10 \%$ of patients and penile skin lesions have been reported in $1 \%-5 \%$ of patients after PAE (1-8). The slightly higher rate of these 

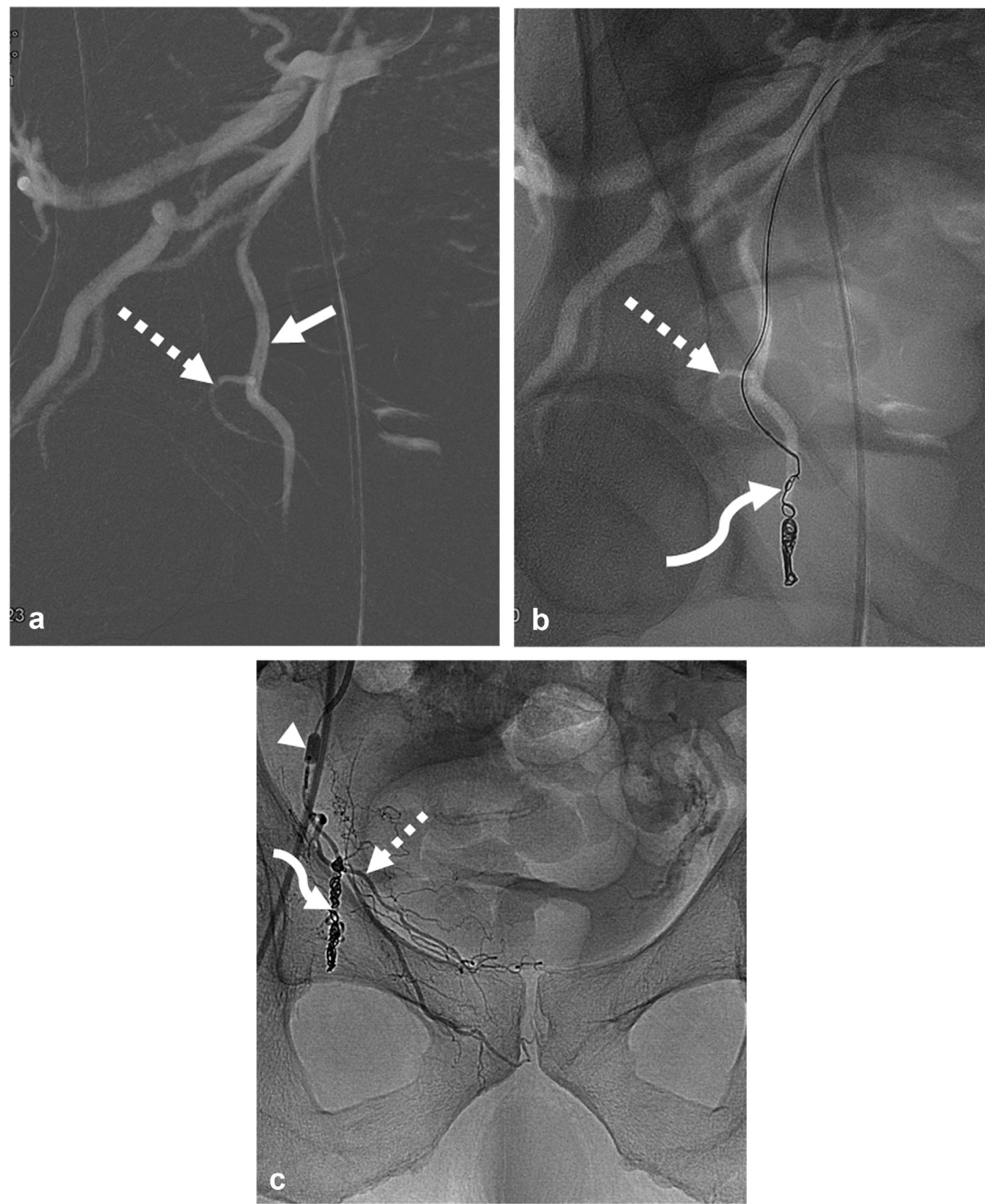

Figure 6. Coil-assisted balloon occlusion right PAE. (a) Roadmap of the right internal iliac artery in ipsilateral anterior oblique view (35 $)$ depicting the right prostatic artery (dashed arrow) arising with an angulated origin from the obturator artery (arrow). (b) Selective catheterization was not possible, so coil embolization of the obturator artery (curved arrow) distally to the right prostatic artery (dashed arrow) was performed. (c) Right prostatic artery digital subtraction angiography in posteroanterior view with the occlusion balloon inflated (arrowhead) depicting the right prostate gland artery (dashed arrow) and the coils occluding the distal obturator artery (curved arrow).

adverse events in the cPAE group could be due to the wedged embolization technique used as opposed to a freeflow embolization and to a low usage of protective coils (14\%). The remaining adverse events, such as irritative voiding, dysuria, hematuria, acute urinary retention, and urinary tract infection, can be considered expectable as a consequence of prostate tissue ischemia. Hematospermia was detected in both groups and, in the bPAE group, was likely due to the proximal position of the microcatheter inside the prostatic artery. Branches to the seminal vesicles, which can arise from the middle/distal third of the prostatic artery, could thus have received inadvertent embolization. Future trials comparing bPAE and cPAE using radiopaque microspheres (10) or macroaggregates (13) could clarify any potential benefits related to reduced nontarget embolization in particular.

The difference in coil usage between groups also failed to show significance. Coils were used more often than anticipated in the bPAE group, in cases where selective catheterization of the prostatic artery was not possible and flow needed to be redirected into the prostate. Also, fewer coils were required than expected in the cPAE group $(14 \%$ of 
Table 2. Within-Group Analysis of Efficacy Variables

\begin{tabular}{|c|c|c|c|c|c|c|}
\hline \multirow[t]{2}{*}{ Variable } & \multicolumn{3}{|c|}{$\operatorname{cPAE}(n=42)$} & \multicolumn{3}{|c|}{ bPAE $(n=42)$} \\
\hline & $\begin{array}{l}\text { Baseline, } \\
\text { Mean (SD) }\end{array}$ & $\begin{array}{c}\text { Last Observation, } \\
\text { Mean (SD) }\end{array}$ & $P$ & $\begin{array}{l}\text { Baseline, } \\
\text { Mean (SD) }\end{array}$ & $\begin{array}{c}\text { Last Observation, } \\
\text { Mean (SD) }\end{array}$ & $P$ Value \\
\hline IPSS & $20.0(6.60)$ & $12.5(8.38)$ & $<.001$ & $20.6(6.67)$ & $12.3(7.93)$ & $<.001$ \\
\hline QoL & $4.74(0.79)$ & $2.60(1.45)$ & $<.001$ & $4.85(0.76)$ & $3.22(1.52)$ & $<.001$ \\
\hline IIEF & $17.5(5.96)$ & $17.8(5.69)$ & .608 & $18.2(5.66)$ & $18.4(5.51)$ & .796 \\
\hline $\mathrm{PV}, \mathrm{cm}^{3}$ & $83.0(56.7)$ & $61.0(29.6)$ & .008 & $71.2(32.0)$ & $65.0(28.8)$ & .006 \\
\hline $\mathrm{PSA}, \mathrm{ng} / \mathrm{mL}$ & $4.00(3.41)$ & $3.10(2.90)$ & .011 & $2.91(2.39)$ & $2.69(2.06)$ & .375 \\
\hline
\end{tabular}

$\mathrm{bPAE}=$ balloon occlusion prostatic artery embolization; $\mathrm{CPAE}=$ conventional microcatheter prostatic artery embolization; IIEF = International Index of Erectile Function; IPSS/QoL = International Prostate Symptom Score/quality of life; PSA = prostate-specific antigen; PV = prostate volume; PVR = postvoid urine residual volume; Omax = peak urinary flow rate.

Table 3. Changes from Baseline

\begin{tabular}{|c|c|c|c|c|c|}
\hline Variable & $\begin{array}{l}\text { cPAE (n = 42), } \\
\text { Mean (SD) }\end{array}$ & $\begin{array}{l}\text { bPAE (n = 42), } \\
\text { Mean (SD) }\end{array}$ & \multicolumn{2}{|c|}{ Difference } & $P$ Value \\
\hline IPSS & $-7.58(6.88)$ & $-8.30(8.12)$ & 0.72 & -2.46 to 3.91 & .653 \\
\hline IIEF & $0.29(3.45)$ & $0.13(3.08)$ & 0.16 & -1.32 to 1.64 & .829 \\
\hline $\mathrm{PV}, \mathrm{cm}^{3}$ & $-21.9(51.6)$ & $-6.15(14.6)$ & -15.8 & -31.5 to -0.01 & .050 \\
\hline Omax, mL/s & $1.85(5.49)$ & $3.12(4.42)$ & -1.26 & -3.55 to 1.03 & .276 \\
\hline $\mathrm{PSA}, \mathrm{ng} / \mathrm{mL}$ & $-0.90(2.22)$ & $-0.22(1.65)$ & -0.68 & -1.50 to 0.14 & .102 \\
\hline
\end{tabular}

$\mathrm{bPAE}=$ balloon occlusion prostatic artery embolization; $\mathrm{Cl}=$ confidence interval; $\mathrm{cPAE}=$ conventional microcatheter prostatic artery embolization; IIEF = International Index of Erectile Function; IPSS/QoL = International Prostate Symptom Score/quality of life; PSA = prostate-specific antigen; $\mathrm{PV}=$ prostate volume; $\mathrm{PVR}=$ postvoid urine residual volume; $\mathrm{Qmax}=$ peak urinary flow rate.

patients). In contrast to the bPAE group, coils were placed in the cPAE group to block anastomoses in the majority of patients. Other studies reported using coils with cPAE in $20 \%-26 \%$ of patients $(8,14,15)$. Because the placement of coils has been shown to increase procedural times and radiation exposure (8), their use should be carefully considered.

Another potential advantage of bPAE would be the establishment of an intraprostate negative pressure that could likely allow the use of a larger amount of embolic volume. If so, bPAE could lead to greater prostatic destruction, together with higher values of PSA in the 24 hours following embolization and greater clinical relief. This was not proven here, as not only was the embolic volume similar between bPAE and cPAE, but also the PSA values and IPSS improvements were not different between the 2 groups. In addition, the volume of embolic material used failed to correlate with IPSS improvement. Any clinical benefits arising from the use of larger amounts of embolic material did not prove to be significant. Previous studies have shown that $5 \alpha$-reductase inhibitors were not detrimental to the clinical outcomes after PAE (16). The present study not only corroborated those findings but also uncovered a potential positive interaction with PAE outcomes - that is, the use of $5 \alpha$-reductase inhibitors before PAE may lead to better clinical outcomes.

This study has some limitations. Differences between the 2 groups in most outcome measures were smaller than initially anticipated, with large variability in the data causing the sample size to be too small to show potential statistically significant differences even with 50 subjects per group. Nevertheless, these results are relevant and may contribute to a clearer understanding of several decisive PAE aspects to be addressed in future systematic reviews and metaanalyses. The outcome measures were assessed only in the short-term, at 6 months, but it is common for most clinical failures after PAE to occur immediately in patients who are nonresponders. The goal of the present study was to assess whether this modified technique could lead to better outcomes. As noted, no significant differences were noted. Long-term data will be needed to understand if the relapse rate is lower with bPAE. Validated questionnaires were not used to quantify the presence and severity of ejaculatory dysfunction and incontinence before or after treatment. Data on pressure measurements, imageable beads, and magnetic resonance imaging after PAE were not available, limiting the assessment of nontarget embolization. The wedged embolization technique in the cPAE group has not been 
Table 4. Adverse Events

\begin{tabular}{lccc} 
Description & cPAE $(\mathbf{n}=\mathbf{4 2}), \mathbf{b P A E}(\mathbf{n}=\mathbf{4 2}), \boldsymbol{P}$ Value \\
& $\mathbf{n}(\mathbf{\%})$ & $\mathbf{n}(\mathbf{\%})$ & \\
Irritative voiding & $19(44.2)$ & $21(45.7)$ & $>.99$ \\
Dysuria & $16(37.2)$ & $17(37.0)$ & $>.99$ \\
Penile skin lesions & $3(6.98)$ & $0(0.0)$ & .109 \\
Hematuria & $2(4.65)$ & $3(6.52)$ & $>.99$ \\
Rectal bleeding & $2(4.65)$ & $0(0.0)$ & .231 \\
Acute urinary retention & $1(2.33)$ & $0(0.0)$ & .489 \\
Hematospermia & $1(2.33)$ & $2(4.35)$ & $>.99$ \\
Groin hematoma & $1(2.33)$ & $0(0.0)$ & .489 \\
UTI & $1(2.33)$ & $2(4.35)$ & $>.99$ \\
Total & $23(53.5)$ & $26(56.5)$ & .833 \\
\hline
\end{tabular}

bPAE = balloon occlusion prostatic artery embolization; cPAE $=$ conventional microcatheter prostatic artery embolization; UTI = urinary tract infection.

previously compared with other embolization techniques of the prostate and could limit the differences with the bPAE group. This wedged embolization technique was applied because premature stasis can be obtained during free-flow PAE with spherical embolic agents leading to low volumes of embolic material used. With the technique of the cPAE group, there was a potential to use larger volumes of embolic material, which was believed to lead to better clinical outcomes. These assumptions failed to be proven in the present study. Future trials comparing free flow with wedged embolization techniques are warranted. Clinical outcome measures after PAE improved less than previously reported (1-8), which can be due to different cohorts being analyzed, different inclusion and exclusion criteria, different follow-up time points, or variations of the PAE techniques being tested. The use of 2.4-F microcatheters could also be arguably considered too large for PAE. Future trials comparing PAE with 2.4-F and smaller microcatheters will be needed to test this hypothesis.

In conclusion, bPAE is safe and effective in the treatment of patients with benign prostatic hyperplasia, with no evidence of clinically relevant differences in outcome measures compared with cPAE. A potential to reduce nontarget embolization to the penis or rectum was noted with bPAE.

\section{REFERENCES}

1. Uflacker A, Haskal ZJ, Bilhim T, Patrie J, Huber T, Pisco JM. Metaanalysis of prostatic artery embolization for benign prostatic hyperplasia. J Vasc Interv Radiol 2016; 27:1686-1697.e8.

2. Pisco JM, Bilhim T, Pinheiro LC, et al. Medium- and long-term outcome of prostate artery embolization for patients with benign prostatic hyperplasia: results in 630 patients. J Vasc Interv Radiol 2016; 27:1115-1122.

3. Bilhim T, Pisco J, Pereira JA, et al. Predictors of clinical outcome after prostate artery embolization with spherical and nonspherical polyvinyl alcohol particles in patients with benign prostatic hyperplasia. Radiology 2016; 281:289-300.

4. Carnevale FC, Iscaife A, Yoshinaga EM, Moreira AM, Antunes AA, Srougi M Transurethral resection of the prostate (TURP) versus original and PErFecTED prostate artery embolization (PAE) due to benign prostatic hyperplasia (BPH): preliminary results of a single center, prospective, urodynamiccontrolled analysis. Cardiovasc Intervent Radiol 2016; 39:44-52.

5. Bilhim T, Pisco J, Campos Pinheiro L, et al. Does polyvinyl alcohol particle size change the outcome of prostatic arterial embolization for benign prostatic hyperplasia? Results from a single-center randomized prospective study. J Vasc Interv Radiol 2013; 24:1595-1602.e1.

6. Gonçalves OM, Carnevale FC, Moreira AM, Antunes AA, Rodrigues VC, Srougi M. Comparative study using 100-300 versus 300-500 $\mu \mathrm{m}$ microspheres for symptomatic patients due to enlarged-BPH prostates. Cardiovasc Intervent Radiol 2016; 39:1372-1378.

7. Wang $\mathrm{MQ}$, Zhang $\mathrm{JL}$, Xin $\mathrm{HN}$, et al. Comparison of clinical outcomes of prostatic artery embolization with $50-\mu \mathrm{m}$ plus $100-\mu \mathrm{m}$ polyvinyl alcohol (PVA) particles versus 100- $\mu$ m PVA particles alone: a prospective randomized trial. J Vasc Interv Radiol 2018; 29:1694-1702.

8. Hacking N, Vigneswaran G, Maclean D, et al. Technical and imaging outcomes from the UK Registry of Prostate Artery Embolization (UKROPE) study: focusing on predictors of clinical success. Cardiovasc Intervent Radiol 2019; 42:666-676.

9. Bilhim T. Endovascular resection of the prostate: how much is enough for prostate artery embolization? J Vasc Interv Radiol 2019; 30:225-227.

10. Isaacson AJ, Hartman TS, Bagla S, Burke CT. Initial experience with balloon-occlusion prostatic artery embolization. J Vasc Interv Radiol 2018; 29:85-89.

11. Bilhim T, Pisco JM, Rio Tinto $H$, et al. Prostatic arterial supply: anatomic and imaging findings relevant for selective arterial embolization. J Vasc Interv Radiol 2012; 23:1403-1415.

12. Angle JF, Siddigi NH, Wallace MJ, et al. Quality improvement guidelines for percutaneous transcatheter embolization: Society of Interventional Radiology Standards of Practice Committee. J Vasc Interv Radiol 2010; 21:1479-1486.

13. Abele JT, Moore R, Tymchak W, Owen RJ. Prostate perfusion mapped by technetium-99m macroaggregated albumin after selective arterial injection. J Vasc Interv Radiol 2015; 26:418-425.

14. Bhatia S, Sinha V, Bordegaray M, Kably I, Harward S, Narayanan G. Role of coil embolization during prostatic artery embolization: incidence, indications, and safety profile. J Vasc Interv Radiol 2017; 28:656-664.e3.

15. Amouyal G, Chague P, Pellerin O, et al. Safety and efficacy of occlusion of large extra-prostatic anastomoses during prostatic artery embolization for symptomatic BPH. Cardiovasc Intervent Radiol 2016; 39:1245-1255.

16. Cardarelli-Leite L, de Assis AM, Moreira AM, et al. Impact of 5-alphareductase inhibitors use at the time of prostatic artery embolization for treatment of benign prostatic obstruction. J Vasc Interv Radiol 2019; 30: 228-232. 